\title{
A utilização do óxido nítrico inalado em cirurgia cardíaca: atualização e análise crítica
}

\author{
Paulo Roberto B. ÉVORA, Fernanda VIARO, Maurício Shigeo OSHIRO, Daniel Gonçalves de SOUZA
}

Laboratório de Função Endotelial da Divisão de Cirurgia Experimental do Departamento de Cirurgia e Anatomia - Faculdade de Medicina de Ribeirão Preto - USP. CECORP - Centro Especializado do Coração e Pulmão de Ribeirão Preto.

RBCCV 44205-593

\section{Resumo}

Objetivo: Apresentar uma revisão e análise crítica sobre a utilização do óxido nítrico (NO) pela via inalatória em cirurgia cardíaca.

Método: metanálise de artigos publicados e inseridos no banco de dados MEDLINE da National Library of Medicine dos Estados Unidos da América. Foram enfatizados aspectos da biologia do NO, mecanismos de ação seletiva do NO pela via inalatória, aspectos técnicos e éticos, aplicações clínicas em cirurgia cardíaca, além de uma análise crítica procurando demonstrar a atual posição da utilização do NO pela via inalatória.

Resultado: As principais evidências foram: a) O NO inalado é reconhecido, atualmente, como um valioso recurso farmacológico da medicina intensiva neonatal e pediátrica, e para a cirurgia cardiopulmonar; b) Outras aplicações em adultos, como a doença pulmonar obstrutiva crônica e a síndrome da angústia respiratória do adulto, necessitam de cuidadosa observação; c) A terapêutica com o NO inalado é relativamente barata, mas não deve ser utilizada em todos os pacientes, com base nos paradigmas de sua eficiência e potencial toxicidade; d) As recentes descobertas de seus efeitos antiinflamatórios e extrapulmonares abrem novos horizontes para futuras aplicações.

Conclusão: Embora seja evidente a extrema variabilidade da resposta vasodilatadora pulmonar seletiva do NO inalado, serviços que tratam pacientes portadores de distúrbios respiratórios, principalmente associados à hipertensão pulmonar, devem dispor do NO inalado como recurso terapêutico. Na ausência de ensaios envolvendo grande número de pacientes, e, apesar de seu potencial tóxico, o NO inalado deve ser utilizado com extremo rigor técnico, como uma prova terapêutica que pode salvar vidas.

Descritores: Óxido nítrico, uso terapêutico. Hipertensão pulmonar. Procedimentos cirúrgicos cardíacos.

\section{Abstract}

Purpose: To present a literature review and critical analysis about the inhaled nitric oxide (NO) application in heart surgery.

Method: metaanalysis of published papers indexed in the MEDLINE database of the United States National Library of Medicine. Points emphasized were: the NO biology, mechanism and selective site of the inhaled NO, technical and ethical aspects, clinical applications in heart surgery. Also, a critical analysis, attempt to demonstrate the current concepts about the inhaled NO therapeutical applications

Result: The main evidences were: the) Inhaled NO is presently recognized as a valuable pharmacological tool in the milieu of the neonatal and pediatric intensive medicine and cardiothoracic surgery; b) Other adult applications, as obstructive lung disease and acute respiratory distress syndrome, need careful observations; c) The inhaled NO therapeutics is relatively inexpensive, but it is important to consider that it should not be used in all patients, based on paradigms of efficiency and potential toxicity; d) The recent discoveries of their anti-inflammatory effects and non pulmonary effects open new horizons for future applications.

Conclusion: Although it is evident the extreme variability of the inhaled NO pulmonary vasodilatatory selective effect, medical centers that treat patients with cardiopulmonary diseases, mainly associate to pulmonary hypertension, owe disposal of this therapeutic resource. In the absence of trials involving great number of patients, and, in spite of its potential toxicity, the inhaled NO therapeutics have to be used under rigid technical protocols to save lives.

Descriptors: Nitric oxide, therapeutic use. Pulmonary hypertension. Cardiac surgical procedures.

Apoio FAPESP - Fundação de Amparo a Pesquisa do Estado de São Paulo.

Endereço para correspondência: Paulo Roberto B. Évora

Rua Rui Barbosa, 367, Ap. 15 - 14015-120 - Ribeirão Preto, SP

E-mail: prbevora@keynet.com.br 


\section{INTRODUÇÃO}

A simples verificação de que a acetilcolina só age como vasodilatador na presença do endotélio desencadeou uma era de intensos trabalhos na década de 80 , a qual estabeleceu o endotélio como sede do início da maioria das doenças cardiovasculares. Postulou-se, então, a existência de um fator relaxante derivado do endotélio ${ }^{(1)}$, que em 1982 foi denominado de "endothelium-derived relaxing factor" $\mathrm{EDRF}^{(2)}$. Utilizando-se cultura de células endoteliais, descobriu-se que esse relaxamento dependente do endotélio associava-se a uma elevação do GMP cíclico na musculatura lisa vascular, podendo ser inibido pelo azul de metileno (GMP cíclico) e pela hemoglobina ("seqüestrador" ou "scavenger" do EDRF) ${ }^{(3)}$. Com o acúmulo de evidências de que o EDRF apresentava características semelhantes aos nitrovasodilatadores, FURCHGOTT E IGNARRO ${ }^{(4,5)}$ propuseram, independentemente, que o EDRF era o óxido nítrico (nitric oxide - NO). As pesquisas direcionaram-se, então, no sentido de determinar-se como o endotélio produz o radical, e culminaram com a proposição de PALMER E MONCADA $^{(6,7)}$ que postularam ser a L-arginina a fonte de NO sob a ação de uma enzima, a óxido nítrico sintase.

A idéia de que o NO inalado poderia causar vasodilatação pulmonar seletiva motivou estudos em animais onde a hipertensão pulmonar era produzida por diversos mecanismos ${ }^{(8-11)}$. Estes estudos demonstraram que concentrações de 5 a $80 \mathrm{ppm}$ produziam vasodilatação pulmonar rápida e reversível, sem efeitos sistêmicos ou reações adversas. No pulmão, como em qualquer outra parte do corpo, o NO formado a partir da L-arginina em uma reação catalisada pela NO-sintase induz vasodilatação através de uma via dependente do GMPc. Uma vez que o NO existe como um gás ele pode ser ministrado por inalação para os alvéolos e vasos sangüíneos próximos a estes alvéolos. Como sofre rápida inativação pela hemoglobina, o NO inalado consegue realizar uma vasodilatação pulmonar seletiva quando existe uma vasoconstrição pulmonar, sem causar vasodilatação sistêmica.

\section{Mecanismos de ação}

A existência de um tono vascular dependente do $\mathrm{NO}$ levou à demonstração de que sua remoção, com consequiente "up?regulation" dos receptores ligados à via de liberação do NO, resulta no aumento da sensibilidade a vasodilatadores que atuam através desta via.

O leito vascular pulmonar apresenta caracteristicamente uma baixa resistência ao fluxo. A hipertensão neste território pode ser devida a uma obstrução pós?capilar ou a um aumento do fluxo para este sistema. Quando persiste por muito tempo este quadro leva a alterações secundárias nestes vasos onde se observa proliferação da camada muscular, fibrose e obliteração da luz. Este quadro, já irreversível, associa-se a uma elevada morbimortalidade. Correções cirúrgicas de cardiopatias presentes como causa de base devem ser tentadas enquanto o sistema vascular pulmonar ainda é responsivo. Uma avaliação pré?operatória desta responsividade pode ser possível com a inalação de
NO. O uso de vasodilatadores sistêmicos pode trazer complicações indesejadas como a hipotensão arterial sistêmica, agravamento de um shunt direita?esquerda e até uma subestimativa do real potencial de vasodilatação que o leito pulmonar oferece. Da mesma forma, a inalação de NO pode ser importante no manejo de situações de difícil controle como o vasoespasmo pulmonar observado no pósoperatório de algumas cirurgias cardíacas, reduzindo o trabalho imposto sobre o ventrículo direito e melhorando a oxigenação.

Pela rápida inativação causada pela hemoglobina e seu curto tempo de meia-vida, o NO inalado deve ativar vasodilatação pulmonar seletiva quando existir uma vasoconstrição, secundária a uma disfunção endotelial ou como um resultado de uma abundante influência vasoconstritora $^{(12-14)}$.

O NO deve permitir uma melhor oxigenação quando administrado na presença de uma perfusão e ventilação equilibradas. Então, têm-se vantagens sobre vasodilatadores administrados endogenamente que causam hipotensão e aumentam o "shunt" intrapulmonar.

A efetividade do NO inalado como um vasodilador pulmonar, em pacientes onde o dano endotelial está associado ao estado da doença, aumenta a questão quanto à deficiência do NO lançado endogenamente ser responsável pela elevação do tônus vascular pulmonar ${ }^{(13)}$. O NO seria lançado continuamente abaixo das condições basais e a inibição destas condições de liberação basal poderia conduzir a um aumento da resistência vascular ${ }^{(15)}$. A perfusão dos pulmões humanos isoladamente com azul de metileno, um inibidor do vasorelaxamento mediado pelo NO, leva a um aumento na resistência vascular pulmonar ${ }^{(16)}$. Desta forma, danos endoteliais, com redução de NO endovenoso, devem ser considerados quando a vasoconstrição pulmonar é conseqüente a doença (ex. SARA - Síndrome da Angústia Respiratória em Adultos) ou a um efeito colateral passageiro do tratamento (ex. "bypass" cardiopulmonar).

O NO inalado, diferente do NO intravenoso, tem ação limitada a veias e artérias de pequenas resistências, e é impossibilitado de dilatar vasos de grande capacidade ${ }^{(17)}$. Nos pulmões perfundidos com NO inalado afeta-se primariamente os vasos arteriais, mas durante extrema vasoconstrição venosa pode agir, também, em nível póscapilar. Em adultos com doença pulmonar aguda, o NO tem um efeito predominantemente vasodilatador na vascularização pulmonar venosa ${ }^{(18)}$. Este aumento na responsividade aparece em pacientes pediátricos com hipertensão pulmonar venosa, nos quais o NO deve resultar em vasodilatação com uma combinação de vasos pré e póscapilares.

A ocorrência de insuficiência ventricular direita secundária à hipertensão arterial pulmonar é a principal complicação pós-operatória de cirurgia cardíaca em crianças e adultos. A vasodilatação pulmonar seletiva produzida pelo NO inalado constitui uma opção terapêutica que, em determinadas situações, pode ser fundamental na administração desta afecção. O NO liga-se à hemoglobina, resultando em sua inativação imediata. Isto resulta em 
manutenção das pressões sangüíneas sistêmicas e coronárias (Figura 1).

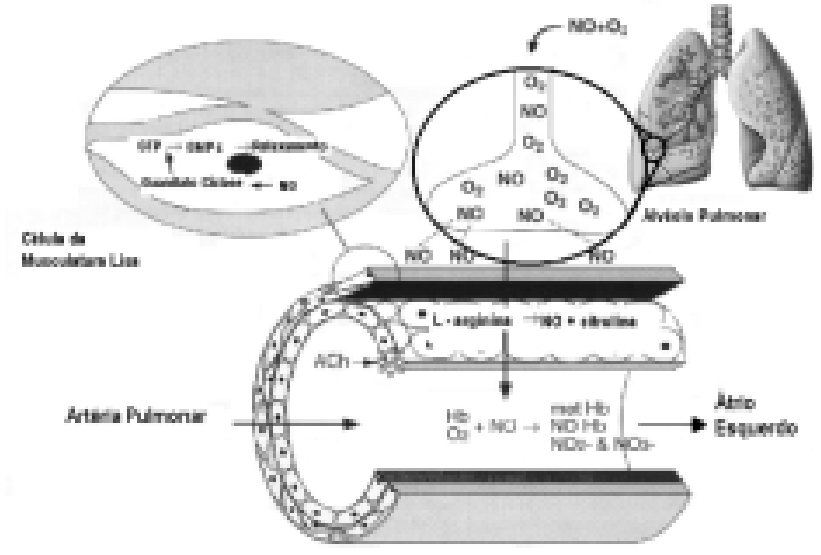

Owdo ntico NOS t endogenarerte fornado per L-aysina depus we a aceticolina (Ach)

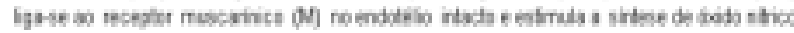

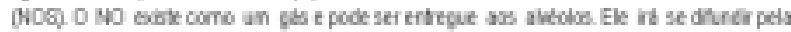

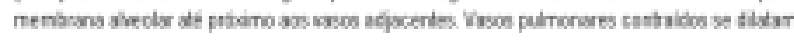

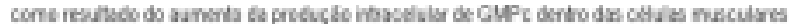

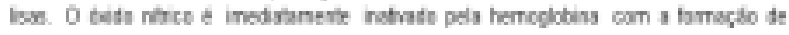

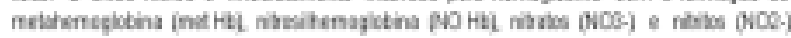
intendo sua atridsde a crrulaçlo pul nons.

Fig. 1 - Representação esquemática do mecanismo e local de ação do öxido nítrico inalado (Adaptado de Atz e Wessel ${ }^{(46)}$ )

Em lesões pulmonares agudas, o NO inalado é preferencialmente liberado em áreas onde a ventilação é alta. Vasos sangüíneos são afetados pela vasoconstrição hipóxica na proximidade de alvéolos mal ventilados. O NO inalado, nestas condições, redireciona o fluxo sangüíneo pulmonar para vasos dilatados próximos a alvéolos bem ventilados, diminui o shunt intrapulmonar e melhora a oxigenação (Figura 2).

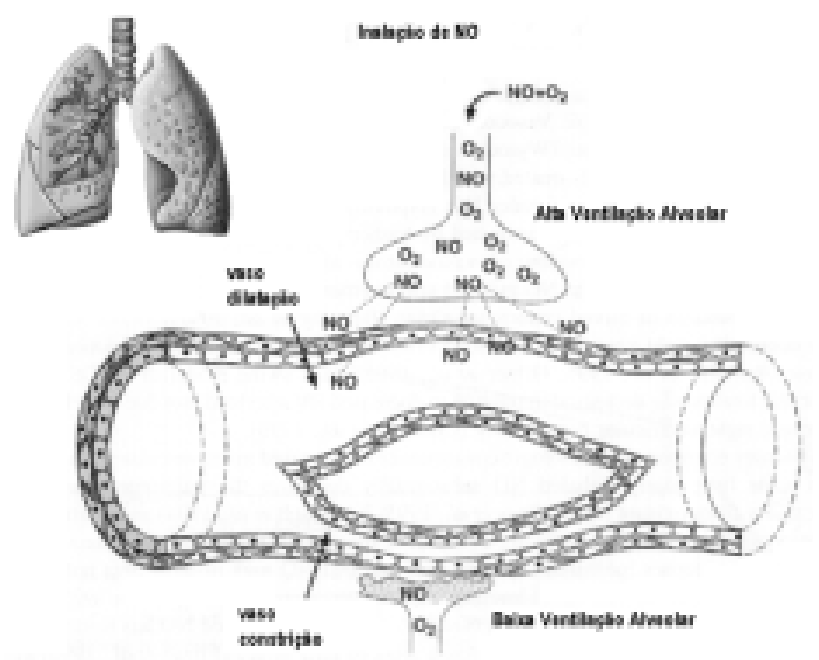

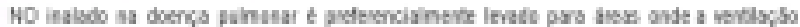

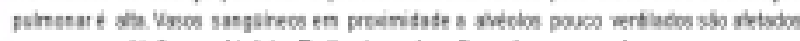

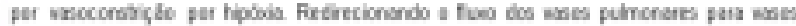

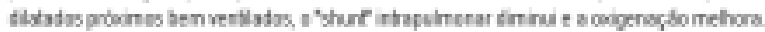

Fig. 2 - Representação esquemática do mecanismo de ação na doença pulmonar (Ex. SARA) (Adaptado de Atz e Wessel ${ }^{(46)}$ )
Um estudo bem delineado no INCOR em 14 pacientes adultos com hipertensão pulmonar confirmou a ação do NO inalado, porém com a ressalva de que a mecânica pulmonar pode interferir na sua eficácia ${ }^{(19)}$.

\section{Aspectos técnicos e éticos}

O NO é tóxico quando inalado em níveis elevados. Ele produz metahemoglobinemia e lesão pulmonar primariamente pela oxidação a NO2. Experiências internacionais têm mostrado importante vasodilatação pulmonar em pacientes respirando baixas concentrações de 5 - 40 ppm, níveis estes que parecem não serem tóxicos. A utilização de 80 ppm por 3 horas manteve os níveis de metahemoglobina abaixo de $3 \%$. Casos isolados, referidos na literatura evidenciaram que a utilização do $\mathrm{NO}$ por inalação até 53 dias não se associou com níveis elevados de metahemoglobina. O NO não pode ser empregado pela via endovenosa por ser rapidamente inativado pela hemoglobina. Este aspecto torna a via inalatória de emprego bastante seguro para o paciente, uma vez que possíveis excessos do gás absorvido são "seqüestrados" pela hemoglobina. Entretanto, há subgrupos populacionais portadores de alterações da capacidade de redução da metahemoglobina intra-eritrocitária. A possibilidade de estes grupos serem mais vulneráveis à metahemoglobinemia, quando expostos à NO, alerta para os riscos de extrapolações de dados obtidos na Europa ou EUA para populações potencialmente distintas.

As maiores preocupações quanto ao emprego clínico do NO dizem respeito à segurança do pessoal médico $\mathrm{e}$ paramédico envolvido no tratamento do paciente, devido aos efeitos tóxicos do NO2. O U.S. Occupational Safety and Health Administration (O.S.H.A.) estabelece que inalação acima de 25 ppm de NO é permissível dentro de um ambiente de trabalho acima de 8 horas por dia com aumentos periódicos de até $100 \mathrm{ppm}^{(20)}$. Algumas medidas de segurança já estão estabelecidas: 1 - Utilizar cilindros de alumínio na proporção 400-500 ppm de NO completadas com N2, o que diminui a chance de efeitos tóxicos, mesmo em caso de vazamento completo do gás por defeito de válvulas; 2 Utilizar cal sodada na linha inspiratória do respirador para neutralização do NO2; 3 - Administrar concentrações baixas de NO (< 80 ppm); 4 - Não usar ar comprimido (úmido) da rede hospitalar na pré-diluição porque a reação do $\mathrm{NO} 2 \mathrm{com}$ a água forma ácido nítrico que é extremamente corrosivo; 5 - Acrescentar o NO no circuito anterior ao "blender" do ventilador (a introdução no ramo inspiratório traz incertezas e riscos à mistura); 6 - Não fabricar seu próprio NO para uso clínico.

Como toda nova modalidade terapêutica, o uso clínico do NO por via inalatória deve ser aprovado pelos comitês de ética médica. Os resultados preliminares são promissores, mas possíveis efeitos colaterais a curto e longo prazo necessitam avaliação pelo acompanhamento dos pacientes submetidos a este tratamento. Por exemplo, sabe-se que o NO é utilizado como índice de poluição do ar ambiente, não se conhecendo seus efeitos quanto à carcinogênese. Não 
se sabe se os efeitos benéficos na fase aguda são permanentes ou se deixam seqüelas. Do ponto de vista bioquímico, sabe-se que o NO pode reagir com radicais superóxidos formando o ânion peróxinitrito. A decomposição deste ânion leva à formação de um potente oxidante com características do radical hidroxila que é tóxico. De qualquer forma, a aplicação clínica justifica-se pela alta mortalidade das doenças envolvidas. O que não se justifica é o uso indiscriminado antes que se estabeleça a sua real eficiência e segurança.

O mínimo que se exige para a sua utilização clínica inclui: 1 - Cilindros de alumínio com 400-500 ppm/N2; 2 - Válvulas de conexão que garantam a integridade do respirador; 3 Disponibilidade de dosagem de metahemoglobina; 4 Aparelho de quimiluminescência para dosagem, à beira do leito de $\mathrm{NO}$ e NO2; 5 - Circuito especial para o NO uma vez que é corrosivo. A fase experimental exige a monitorização hemodinâmica à beira do leito e pessoal treinado para o projeto.

Além da metahemoglobinemia, dos possíveis efeitos tóxicos do NO2 e do peróxinitrito, além de uma possível associação com carcinogênese, três outros efeitos adversos devem ser considerados: uma possível piora de disfunção ventricular esquerda, um rebote da hipertensão pulmonar ao se suspender a inalação do $\mathrm{NO}$ e a possibilidade de coagulopatia pelos efeitos antiplaquetários do NO.

Experiência clínica com o NO inalado em cirurgia cardíaca

\section{Cardiopatias congênitas}

O NO inalado é um potente vasodilatador seletivo em casos de hipertensão arterial pulmonar, reduzindo graves hipoxemias em neonatos e crianças. Embora os efeitos imediatos possam ser impressionantes, o seguimento em longo prazo quanto à hipertensão pulmonar residual e outras sequielas tem sido motivo de estudos em poucos pacientes. Como um vasodilatador pulmonar seletivo, o NO inalado é um importante agente diagnóstico e terapêutico. O NO inalado pode ser utilizado na prevenção e tratamento de crises hipertensivas pulmonares após circulação extracorpórea (CEC). Entretanto, até o momento, não existe nenhum estudo randomizado comprovando os benefícios do NO inalado em crianças com cardiopatia congênita ${ }^{(21)}$. Um estudo duplo-cego envolvendo 124 crianças com idade média de três meses, portadoras de CIVs e DSAVs concluiu que em crianças com alto risco de hipertensão pulmonar, o uso rotineiro de $\mathrm{NO}$ inalado após cirurgia corretiva pode, sem nenhum efeito tóxico, diminuir o risco de crises hipertensivas pulmonares e encurtar o período pósoperatório ${ }^{(22)}$. Em um estudo incluindo 40 pacientes, o NO não melhorou, substancialmente, a hemodinâmica pulmonar e trocas gasosas logo após cirurgias de cardiopatias congênitas. Neste estudo, o NO também falhou em reduzir, significantemente, a incidência de crises hipertensivas pulmonares ${ }^{(23)}$.

Um potencial do NO inalado poderia ser a sua utilização em crianças submetidas a cirurgia cardíaca, as quais não podem ser liberadas da CEC. Nestas condições, o NO inalado reduz, seletivamente, a pressão média na artéria pulmonar (PAPm) em crianças que saem de CEC com hipertensão pulmonar, e, não tem nenhum efeito quando não ocorre este tipo de hipertensão. Em um estudo duplo-cego randomizado, o NO inalado reduziu seletivamente as pressões em artéria pulmonar, em pacientes pediátricos que desenvolvem hipertensão pulmonar, imediatamente após CEC e correção cirúrgica $^{(24)}$.

Como um vasodilatador pulmonar seletivo, o NO inalado é um importante agente diagnóstico e terapêutico. $\mathrm{Na}$ tentativa de definir-se subgrupos que poderiam ser beneficiados, em uma experiência com 400 pacientes tratados com NO inalado, sendo $37 \%$ neonatos, benefícios foram obtidos em neonatos com drenagem anômala total de veias pulmonares, estenose mitral congênita, e em pacientes com shunts esquerda-direita ${ }^{(21)}$. Como recurso diagnóstico o NO inalado diferencia o vasoespasmo pulmonar da obstrução vascular após cirurgia para correção de cardiopatias congênitas ${ }^{(25)}$. Um ensaio sobre a sua utilização após cirurgia cardíaca em neonatos e crianças seria útil para o estabelecimento de parâmetros de diferenciação entre vasoconstrição pulmonar reversível e obstruções anatômicas fixas. Estas informações poderiam, inclusive, suportar a indicação de suporte com a utilização de oxigenação extracorpórea de membrana (ECMO). Assim, a não resposta ao NO inalado indicaria a necessidade de investigações adicionais ${ }^{(26)}$.

É curiosa a constatação de que o tratamento com NO inalado possa resultar em diminuição da resistência vascular pulmonar e melhorar a ejeção ecocardiográfica do ventrículo direito sem, necessariamente, induzir alterações significantes da pressão na artéria pulmonar ${ }^{(27)}$.

\section{Cardiopatias adquiridas do adulto}

Um primeiro estudo, em 1989, realizado depois de uma substituição de valva mitral devido à estenose com hipertensão pulmonar, sugeriu a utilização do NO inalado para controlar a hipertensão pulmonar em cirurgia cardíaca. O uso efetivo do NO inalado após cirurgia da valva mitral e revascularização miocárdica tem sido relatado em experiências isoladas, não existindo nenhum grande ensaio. A vasodilatação pulmonar seletiva tem sido demonstrada em pacientes com hipertensão pulmonar secundária à valvopatia mitral. A diminuição da resistência pulmonar, em média, é de $20 \%$, enquanto a diminuição da pressão na artéria pulmonar é de $10 \%$. Interessante estudo relata que a resposta ao NO inalado em pacientes adultos valvopatas é variável. Quando se utiliza o NO inalado em adulto portadores de valvopatia mitral congênita, os efeitos do NO inalado são mais acentuados, provavelmente porque a vasoconstrição nestes pacientes é maior e a musculatura lisa vascular hipertrofiada.

Um estudo prospectivo, randomizado, sem a condição de ser duplo-cego, utilizou o NO inalado em 62 pacientes consecutivos, os quais demonstravam hipertensão 
pulmonar imediatamente antes da indução anestésica. $\mathrm{O}$ objetivo, além de observar a eficácia do NO inalado, foi testar diferentes doses de inalação. Foram testadas as doses de 10, 20, 30 e 40 ppm, sendo um quinto grupo, considerado controle, tratado com um bolo endovenoso de $50 \mathrm{mg} / \mathrm{kg}$ de milrinone e dose de manutenção de $0.5 \mathrm{microg} / \mathrm{kg} / \mathrm{min}$. Não existia nenhuma diferença demográfica entre os grupos e não ocorreu nenhuma diferença significante na porcentagem de diminuição da resistência vascular pulmonar. Este estudo conclui que não se justificam doses maiores do que 10 ppm de NO inalado.

Um outro estudo, envolvendo 42 pacientes adultos submetidos a cirurgia cardíaca com CEC, revelou que os pacientes que receberam $40 \mathrm{ppm}$ de $\mathrm{NO}$ inalado, quando comparados com pacientes que receberam 20 ppm ou apenas milrinone endovenoso, apresentaram menor freqüência cardíaca, maior fração de ejeção do ventrículo direito e uma menor necessidade de agentes vasopressores ${ }^{(28)}$.

Embora existam relatos isolados em cirurgias cardíacas em adultos, a análise das experiências publicadas e o conhecimento de conceituados serviços de cirurgia cardíaca, no Brasil e no exterior, permitem afirmar que a utilização do NO inalado não foi incorporada como rotina, constituindose apenas em um recurso para investigação e para a utilização em situações excepcionais.

Em um estudo prospectivo, randomizado, compararamse os efeitos do NO inalado com os efeitos de vasodilatadores sistêmicos em pacientes adultos com hipertensão pulmonar grave e função ventricular direita preservada. O NO inalado não foi superior à prostaglandina E1 em relação ao índice cardíaco e função ventricular direita. Neste estudo, os autores consideram a potencial toxicidade do NO inalado e postulam uma melhor definição do tipo(s) de paciente(s) que, realmente, seriam beneficiados do ponto de vista risco-benefício ${ }^{(29)}$.

\section{Transplante cardíaco}

A resistência vascular pulmonar aumentada é um fator de risco pré-operatório para transplantes cardíacos, a qual se manifesta por risco maior de insuficiência vascular direita no pós-operatório ${ }^{(30,31)}$. Nestas condições a medicação vasodilatadora é freqüentemente necessária e o NO inalado, pela sua seletividade, pode ser uma excelente opção terapêutica. No tratamento da hipertensão pulmonar após transplante cardíaco e pulmonar os efeitos do NO inalado são semelhantes aos resultados obtidos com a utilização do nitroprussiato de sódio, prostaciclina e prostaglandina E1. Mas, nestas condições, o NO inalado foi o único vasodilatador pulmonar seletivo ${ }^{(32-35)}$.

\section{Análise crítica}

Uma metanálise utilizando informações, extraídas até 1996, dos dois bancos de referências mais importantes (Current Contents e Medline) para a pesquisa da literatura médica, além de dados de congressos especializados, permite enumerar alguns dos principais dados quanto à utilização do NO inalado: a) O NO inalado é reconhecido, atualmente, como um valioso recurso farmacológico da medicina intensiva neonatal e pediátrica, e para a cirurgia cardiopulmonar; b) Outras aplicações em adultos, como a doença pulmonar obstrutiva crônica e a síndrome da angústia respiratória aguda do adulto, necessitam de cuidadosa observação; c) A terapêutica com o NO inalado é, relativamente, barata, mas deve ser utilizada em todos os pacientes, com base nos paradigmas de sua eficiência e potencial toxicidade; d) As recentes descobertas de seus efeitos antiinflamatórios e extrapulmonares abrem novos horizontes para futuras aplicações ${ }^{(36)}$.

Além desta postulação geral, uma análise crítica, embora sem a pretensão de abordar todos os aspectos da utilização do NO inalado em cirurgia cardíaca, deve incluir algumas perguntas e possíveis respostas.

Existem vantagens da utilização do NO inalado sobre a hiperventilação para o controle da hipertensão pulmonar após a correção cirúrgica de cardiopatias congênitas?

Em princípio: sim. O NO inalado e a hiperventilação são ambos efetivos em reduzir a pressão na artéria pulmonar (PAP) e a resistência vascular pulmonar (RVP). Porém, a ação seletiva do NO inalado na circulação pulmonar oferece vantagens sobre a hiperventilação porque a queda do débito cardíaco e o aumento da RVS são indesejáveis neste período $^{(37)}$. Ressalte-se que o NO em oxigênio parece ser um vasodilatador pulmonar mais potente do que o oxigênio sozinho $^{(38)}$.

O NO inalado pode ser eficiente para aumentar a sobrevida em cirurgias de cardiopatias congênitas ou adquiridas com hipertensão pulmonar?

Embora o NO inalado possa reduzir a hipertensão pulmonar, parece que esta ação não se associa a uma maior sobrevida. Um estudo randomizado faz-se necessário para determinar o exato papel do NO inalado na sobrevida de pacientes com hipertensão pulmonar residual após tratamento cirúrgico ${ }^{(39)}$.

Existem subgrupos de cardiopatias congênitas que podem obter melhores benefícios com o uso do NO inalado?

A resposta é não. Estudos demonstram que o NO inalado causa mínimo benefício sobre a PAP ou débito cardíaco (DC) em crianças após a correção do canal atrioventricular ${ }^{(40)}$.

É possível prever a necessidade da utilização do NO inalado no pós-operatório de cardiopatias congênitas e adquiridas?

Alguns fatores já foram associados como preditivos da utilização do NO inalado: a) Idade < 1 ano, Síndrome de Down, hipertensão pulmonar pré-operatória e aumento da resistência vascular pulmonar. Usando um modelo multivariado, baseado nestes fatores, $73 \%$ dos pacientes que utilizaram NO inalado foram identificados. Em um serviço 
que permite o uso irrestrito do NO inalado, $50 \%$ das crianças operadas por cardiopatias congênitas fizeram seu uso ${ }^{(41)}$.

O NO inalado pode ser associado ao uso de prostaciclinas?

Existem algumas controvérsias. A combinação de ambos os vasodilatadores não se mostrou mais potente do que o uso isolado do Iloprost ou do NO inalado, tanto para teste farmacológicos, como para o controle da hipertensão pulmonar ${ }^{(42)}$. O Beraprost parece ser uma alternativa terapêutica ao uso do NO inalado e da tolazolina, para estudar a resposta vasodilatadora pulmonar. $\mathrm{O}$ uso combinado de ambos poderia ser uma alternativa terapêutica, sem grandes complicações no tratamento da hipertensão pulmonar em crianças $^{(43)}$.

Existe algum recurso terapêutico contra a hipertensão pulmonar "rebote" após a suspensão do NO inalado?

O dipiridamol poderia atenuar a hipertensão pulmonar rebote após a suspensão do uso do NO inalado no pósoperatório de cardiopatias congênitas. O dipiridamol pode sustentar as elevações do GMPc induzidas pelo NO inalado. Além disso, a atividade da fosfodiesterase poderia contribuir para a hipertensão pulmonar aguda após a suspensão do NO inalado ${ }^{(44)}$.

Considerando-se o potencial tóxico do NO inalado existem estudos de seguimento a médio e longo prazo de pacientes submetidos ao seu uso terapêutico?

Estes estudos ainda são infreqüentes na literatura. Um estudo japonês, relatando o seguimento de 65 crianças em um período de 2.0 a 4.3 anos (média 3.1 anos), afirma que todas as crianças não mais necessitaram usar oxigênio. Além disso, possíveis efeitos adversos, incluindo a ocorrência de tumores malignos ou inflamação crônica do trato respiratório não foram observados ${ }^{(45)}$.

Além da redução da resistência vascular pulmonar existem outros efeitos do NO inalado que podem vir a ser avaliados do ponto de vista terapêutico?

Outras ações do NO inalado devem aumentar o interesse no seu potencial terapêutico: O NO inalado atenua a proliferação da musculatura lisa vascular, inibe a agregação plaquetária, promove citoproteção para órgãos doadores, melhora os aspectos prejudiciais dos danos da reperfusão isquêmica, deve promover angiogênese em pulmões imaturos e melhorar a capacidade de carrear oxigênio pela hemoglobina nas anemias falciformes ${ }^{(46)}$.

Seria possível ou haveria lógica na utilização de drogas doadoras de NO pela via inalatória como uma opção ao NO inalado?

Pelo menos um trabalho publicado indica que a nebulização de nitroglicerina é efetiva, barata e segura para controlar hipertensão pulmonar associada a cardiopatias congênitas em serviços que não contam com os recursos de oxigenação extracorpórea e/ou NO inalado ${ }^{(47)}$. No CTI do Hospital do Coração de Ribeirão Preto e na UTI Neonatal do Hospital da Clínicas de Ribeirão Preto, o nitroprussiato de sódio pela via inalatória já foi utilizado em casos isolados e desesperadores. De maneira completamente aleatória alguns neonatos apresentaram, transitoriamente, vasodilatação e tornaram-se corados. Em alguns casos nenhum efeito foi observado. O nitroprussiato de sódio foi utilizado em uma paciente adulta portadora de grave disfunção ventricular direita secundária à hipertensão pulmonar associada a comunicação interatrial e tromboembolismo pulmonar. No pós-operatório da tromboendarterectomia pulmonar, sete anos após a correção da CIA, os níveis de hipertensão pulmonar alcançaram 180 $\mathrm{mmHg}$. O nitroprussiato de sódio pela via venosa diminuía a pressão arterial pulmonar, mas associava-se a grave hipotensão arterial sistêmica. Quando se tentou a via inalatória a pressão arterial cedia, porém o mesmo ocorria com a pressão sistêmica, ainda que em magnitude menor do que a via venosa. Não foi possível concluir se a hipotensão arterial sistêmica estava associada à função cardíaca ou se havia absorção sistêmica do nitroprussiato utilizado pela via inalatória.

Diante dos aspectos revistos, onde se evidenciam muitas controvérsias, quais seriam as possíveis conclusões? Uma coisa é certa. Embora, comprovadamente, o NO inalado seja um vasodilatador pulmonar seletivo e, sem sombra de dúvidas, venha salvando vidas, a sua utilização não se tornou uma unanimidade em um período superior a 10 anos. Este fato se deve, seguramente, à sua potencial toxicidade e à sua variabilidade de resposta (observada, inclusive, no âmbito da neonatologia geral), fatos estes que não estimulam a realização de grandes ensaios, os quais poderiam esclarecer muitas da mencionadas controvérsias.

No início da década de 90, um dos autores (PRBE) visitou importantes clínicas americanas (Mayo Clinic, Cleveland Clinic, John Hopkins e Harvard), onde se iniciavam as experiências clínicas com o NO inalado. Um resumo das observações e entrevistas nestas clínicas ressaltavam: a) A efetividade do tratamento em casos isolados; b) A variabilidade da resposta terapêutica independente da idade, doença cardíaca e/ou respiratória; c) Melhor resposta terapêutica, ainda que transitória, nos casos de hipertensões pulmonares mais graves e crônicas; d) A crença de que o NO inalado seria uma potencial arma terapêutica em hipertensões transitórias, assim, a neonatologia e os transplantes cardiopulmonares poderiam ser beneficiados e; e) Todos se mostravam apreensivos com a potencial toxicidade do NO, não só para os pacientes, mas também para os profissionais que dele fizessem uso. Parece que em um tempo superior a uma década estas observações ainda são atuais e pertinentes. Apenas um consenso parece estabelecido, qual seja, a utilização de doses menores (10 a $20 \mathrm{ppm}$ ) do que as inicialmente utilizadas.

Uma metanálise das referências citadas no MEDLINE ilustra a evolução e o atual papel do NO inalado na cirurgia cardíaca. Quando se compara o número total de trabalhos publicados com o número de trabalhos em humanos, observa-se que a maioria $(66,6 \%)$ relaciona-se com seres humanos. Quando se associa NO inalado com 
hipertensão pulmonar o número total de trabalhos corresponde a mais de 50\% do total com uma prevalência em torno de $75 \%$ de trabalhos em humanos. Quando se associa o NO inalado como parte do arsenal terapêutico da SARA, o número total de trabalhos corresponde a $12,5 \%$ do total com uma prevalência de cerca de $82 \%$ de trabalhos em humanos (Figura 3).

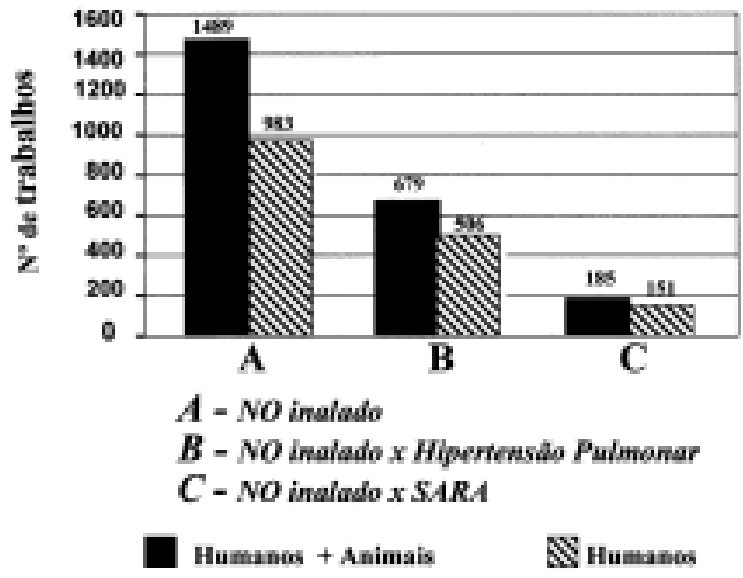

Fig. 3 - Representação gráfica do número de referências obtidas com auxílio do MEDLINE (Dados gerais)

Quando se buscam trabalhos relacionando-se especificamente o uso terapêutico do $\mathrm{NO}$ em cirurgia cardíaca o número de publicações corresponde a cerca de $2,5 \%$ do total. Quando se considera o uso terapêutico do NO inalado não só com a cirurgia cardíaca de um modo geral, mas, também com valvopatias, cardiopatias congênitas, transplante cardíaco e transplante pulmonar, o número total de comunicações está em torno de 200. Destas duzentas publicações, a cirurgia cardíaca em geral corresponde a $1,65 \%$, as valvopatias a $6 \%$, os transplantes cardíacos a $15 \%$, as cardiopatias congênitas a $24 \%$ e os transplantes pulmonares a 35\% (Figura 4) .

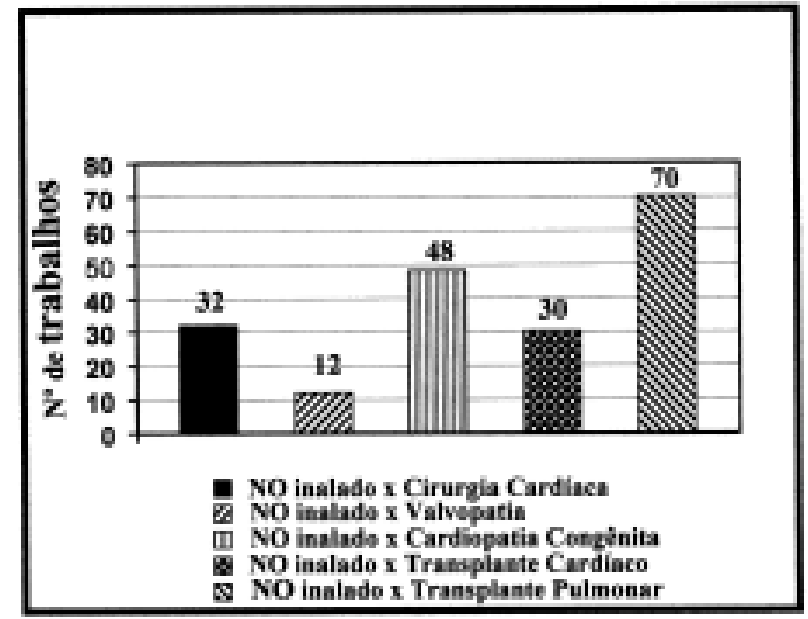

Fig. 4 - Representação gráfica do número de referências obtidas com auxílio do MEDLINE (Dados relativos a cirurgia cardiopulmonar)
Estes dados confirmam as observações quanto ao futuro e tendências da utilização terapêutica do NO inalado. A sua utilização não se tornou unanimidade, sendo os grupos mais beneficiados aqueles em que a hipertensão pulmonar é transitória. É importante notar o relativo pequeno número de relatos, muitos associados a casos isolados, e uma considerável prevalência de observação em humanos sem um grande embasamento experimental. Talvez este fato se deva, em parte, à dificuldade de se obter modelos experimentais de hipertensão pulmonar, mas, certamente à incógnita quanto aos efeitos tóxicos de $\mathrm{NO}$ inalado em longo prazo e à sua variabilidade de resposta efetiva. Um ponto deve ficar claro, serviços que tratam pacientes portadores de distúrbios respiratórios, principalmente associados à hipertensão pulmonar, devem dispor do NO inalado como recurso terapêutico. Na ausência de ensaios envolvendo grande número de pacientes, e, apesar de seu potencial tóxico, o NO inalado deve ser utilizado com extremo rigor técnico, como uma prova terapêutica que pode salvar vidas.

\section{REFERÊNCIAS BIBLIOGRÁFICAS}

1. Furchgott RF \& Zawadzki JV - The obligatory role of endothelial cells in the relaxation of arterial smooth muscle by acetylcholine. Nature 1980; 288: 373 ?6.

2. Cherry PD, Furchgott RF, Zawadzki JV, Jothianandan D Role of endothelial cells in relaxation of isolated arteries by bradykinin. Proc Natl Acad Sci USA 1982; 79:2106-10.

3. Cocks T \& Angus JA - Bioassay of the release of endotheliumderived relaxing factor (EDRF) from isolated endothelial cells in vitro. In: Bevan JA, Godfraind T, Maxwell RA, Stoclet JS, Worcel M, eds. Vascular neuroeffector mechanisms. Amsterdam: Elsevier, 1985: 131-6.

4. Furchgott RF - Studies on relaxation of rabbit aorta by sodium nitrite: the basis for the proposal that the acid-activated inhibitory factor from bovine retractor penis is inorganic nitrite and the endothelium-derived relaxing factor is nitric oxide. In: Vanhoutte PM, ed. Mechanisms of vasodilatation. New York: Raven Press, 1988: 401-14. vol. IV.

5. Ignarro LJ, Byrns RE, Wood KS - Biochemical and pharmacological properties of EDRF and its similarity to nitric oxide radical. In: Vanhoutte PM , ed. Mechanisms of vasodilatation. New York: Raven Press, 1988: 427-35. vol. IV.

6. Palmer RM, Ferrige AG, Moncada S - Nitric oxide release accounts for the biological activity of endothelium-derived relaxing factor. Nature 1987; 327:524-6.

7. Palmer RM, Ashton DS, Moncada S - Vascular endothelial cells synthesize nitric oxide from L-arginine. Nature 1988; 333:664-6. 
8. Frostell C, Fratacci MD, Wain JC, Jones R, Zapol WM Inhaled nitric oxide: a selective pulmonary vasodilator reversing hypoxic pulmonary vasoconstriction. Circulation 1991; 83: $2038 ? 47$.

9. Moncada S, Palmer RM, Higgs EA - Nitric oxide: physiology, pathophysiology, and pharmacology. Pharmacol Rev 1991; 43: 109?42.

10. Snyder SH \& Bredt DS - Biological roles of nitric oxide. Sci Am 1992; 266: 68?77.

11. Tavares AP, Pimenta Junior AG, Evora PR - Basis for the therapeutic use of inhaled nitric oxide. Arq Bras Cardiol 1995; 64:45-52.

12. Frostell CG, Blomqvist H, Hedenstierna G, Lundberg J, Zapol WM - Inhaled nitric oxide selectively reverses human hypoxic pulmonary vasoconstriction without causing systemic vasodilation. Anesthesiology 1993; 78: 427?35.

13. Wessel DL, Adatia I, Giglia TM, Thompson JE, Kulik TJ Use of inhaled nitric oxide and acetylcholine in the evaluation of pulmonary hypertension and endothelial function after cardiopulmonary bypass. Circulation 1993 ; 88(5 pt 1), 212838.

14. Roberts Jr. JD, Lang P, Bigatello LM, Vlahakes GJ, Zapol WM - Inhaled nitric oxide in congenital heart disease. Circulation 1993; 87: 447?53.

15. Vallance P, Collier J, Moncada S - Effects of endotheliumderived nitric oxide on peripheral arteriolar tone in man. Lancet 1989; 2:997-1000.

16. Cremona G, Dinh-Xuan AT, Higenbottam TW - Endotheliumderived relaxing factor and the pulmonary circulation. Lung 1991; 169:185-202.

17. Roos CM, Rich GF, Uncles DR, Daugherty MO, Frank DU Sites of vasodilation by inhaled nitric oxide vs. sodium nitroprusside in endothelin-constricted isolated rat lungs. J Appl Physiol 1994; 77:51-7.

18. Benzing A \& Geiger K - Inhaled nitric oxide lowers pulmonary capillary pressure and changes longitudinal distribution of pulmonary vascular resistance in patients with acute lung injury. Acta Anaesthesiol Scand 1994; 38:640-5.

19. Carmona MJ \& Auler Jr. JO - Effects of inhaled nitric oxide on respiratory system mechanics, hemodynamics, and gas exchange after cardiac surgery. J Cardiothorac Vasc Anesth 1998; 12:157-61.

20. Centers for Disease Control - Recommendation for occupational safety and health standard. MMWR Morb Mortal WKLY Rep 1980; 37: S?7 .
21. Atz AM \& Wessel DL - Inhaled nitric oxide in the neonate with cardiac disease. Semin Perinatol 1997; 21: 441-55.

22. Miller OI, Tang SF, Keech A, Pigott NB, Beller E, Celermajer DS - Inhaled nitric oxide and prevention of pulmonary hypertension after congenital heart surgery: a randomised double-blind study. Lancet 2000; 356:1464-9.

23. Day RW, Hawkins JA, McGough EC, Crezee KL, Orsmond GS - Randomized controlled study of inhaled nitric oxide after operation for congenital heart disease. Ann Thorac Surg 2000; 69:1907-13.

24. Russell IA, Zwass MS, Fineman JR et al. - The effects of inhaled nitric oxide on postoperative pulmonary hypertension in infants and children undergoing surgical repair of congenital heart disease. Anesth Analg 1998; 87: 46-51.

25. Beghetti M, Morris K, Cox P, Bohn D, Adatia I - Inhaled nitric oxide differentiates pulmonary vasospasm from vascular obstruction after surgery for congenital heart disease. Intensive Care Med 1999; 25:1126-30.

26. Wessel DL \& Adatia I - Clinical applications of inhaled nitric oxide in children with pulmonary hypertension. Adv Pharmacol 1995; 34:475-504.

27. Schulze-Neick I, Bultmann M, Werner Het al. - Right ventricular function in patients treated with inhaled nitric oxide after cardiac surgery for congenital heart disease in newborns and children. Am J Cardiol 1997; 80:360-3.

28. Solina A, Papp D, Ginsberg S et al. - A comparison of inhaled nitric oxide and milrinone for the treatment of pulmonary hypertension in adult cardiac surgery patients. J Cardiothorac Vasc Anesth 2000; 14:12-7.

29. Schmid ER, Burki C, Engel MH, Schmidlin D, Tornic M, Seifert B - Inhaled nitric oxide versus intravenous vasodilators in severe pulmonary hypertension after cardiac surgery. Anesth Analg 1999; 89:1108-15.

30. Bando K, Konishi H, Komatsu K et al. - Improved survival following pediatric cardiac transplantation in high-risk patients. Circulation 1993; 88(5 Pt 2):II218-23.

31. Murali S, Kormos RL, Uretsky BF et al. - Preoperative pulmonary hemodynamics and early mortality after orthotopic cardiac transplantation: the Pittsburgh experience. Am Heart J 1993; 126:896-904.

32. Kieler-Jensen N, Lundin S, Ricksten S - Vasodilator therapy after heart transplantation: effects of inhaled nitric oxide and intravenous prostacyclin, prostaglandin E1, and sodium nitroprusside. J Heart Lung Transplant 1995; 14:436-43.

33. Williams TJ, Salamonsen RF, Snell G, Kaye D, Esmore DS Preliminary experience with inhaled nitric oxide for acute pulmonary hypertension after heart transplantation. J Heart Lung Transplant 1995; 14:419-23. 
34. Girard C, Durand PG, Vedrinne C et al. - Inhaled nitric oxide for right ventricular failure after heart transplantation. $\mathrm{J}$ Cardiothorac Vasc Anesth 1993; 7:481-5.

35. Auler Jr. JO, Carmona MJ, Bocchi EA et al. - Low doses of inhaled nitric oxide in heart transplant recipients. J Heart Lung Transplant 1996, 15:443-50.

36. Troncy E, Francoeur M, Blaise G - Inhaled nitric oxide: clinical applications, indications, and toxicology. Can J Anaesth 1997; 44:973-88.

37. Morris K, Beghetti M, Petros A, Adatia I, Bohn D Comparison of hyperventilation and inhaled nitric oxide for pulmonary hypertension after repair of congenital heart disease. Crit Care Med 2000; 28:2974-8.

38. Allman KG, Young JD, Carapiet D, Stevens JE, Ostman-Smith I, Archer LN - Effects of oxygen and nitric oxide in oxygen on pulmonary arterial pressures of children with congenital cardiac defects. Pediatr Cardiol 1996; 17:246-50.

39. Sharma R, Raizada N, Choudhary SK et al. - Does inhaled nitric oxide improve survival in operated congenital disease with severe pulmonary hypertension? Indian Heart J 2001; 53:48-55.

40. Curran RD, Mavroudis C, Backer CL, Sautel M, Zales VR, Wessel DL - Inhaled nitric oxide for children with congenital heart disease and pulmonary hypertension. Ann Thorac Surg 1995; 60:1765-71.
41. Laitinen PO, Rasanen J, Sairanen H - Postoperative nitric oxide therapy in children with congenital heart disease: can the need be predicted? Scand Cardiovasc J 2000; 34:149-53.

42. Rimensberger PC, Spahr-Schopfer I, Berner M et al. - Inhaled nitric oxide versus aerosolized iloprost in secondary pulmonary hypertension in children with congenital heart disease: vasodilator capacity and cellular mechanisms. Circulation 2001; 103:544-8

43. Ichida F, Uese K, Hashimoto I et al. - Acute effect of oral prostacyclin and inhaled nitric oxide on pulmonary hypertension in children. J Cardiol 1997; 29:217-24.

44. Ivy DD, Kinsella JP, Ziegler JW, Abman SH - Dipyridamole attenuates rebound pulmonary hypertension after inhaled nitric oxide withdrawal in postoperative congenital heart disease. $\mathbf{J}$ Thorac Cardiovasc Surg 1998;115:875-82.

45. Yahagi N, Kumon K, Tanigami H et al. - Cardiac surgery and inhaled nitric oxide: indication and follow-up (2-4 years). Artif Organs 1998; 22:886-91.

46. Atz AM \& Wessel DL - Nitric oxide inhalation. In: Rubanyi GM, ed. Pathophysiology and clinical applications of nitric oxide. Netherlands: Harwood Academic Publishers, 1999: 471503.

47. Omar HA, Gong F, Sun MY, Einzig S - Nebulized nitroglycerin in children with pulmonary hypertension secondary to congenital heart disease. W V Med J 1999; 95:74-5. 